\title{
Electrophysiological study of local/global processing in Williams syndrome
}

\author{
Alexandra P. F. Key • Elisabeth M. Dykens
}

Received: 15 March 2010 / Accepted: 23 September 2010 /Published online: 7 October 2010

(C) Springer Science+Business Media, LLC 2010

\begin{abstract}
Persons with Williams syndrome (WS) demonstrate pronounced deficits in visuo-spatial processing. The purpose of the current study was to examine the preferred level of perceptual analysis in young adults with WS $(n=$ 21 ) and the role of attention in the processing of hierarchical stimuli. Navon-like letter stimuli were presented to adults with WS and age-matched typical controls in an oddball paradigm where local and global targets could appear with equal probability. Participants received no explicit instruction to direct their attention toward a particular stimulus level. Behavioral and event-related potential (ERP) data were recorded. Behavioral data indicated presence of a global precedence effect in persons with WS. However, their ERP responses revealed atypical brain mechanisms underlying attention to local information. During the early perceptual analysis, global targets resulted in reduced P1 and enhanced N150 responses in both participant groups. However, only the typical comparison group demonstrated a larger N150 to local targets. At the
\end{abstract}

\footnotetext{
A. P. F. Key $(\bowtie)$

Vanderbilt Kennedy Center for Research on Human Development, Vanderbilt University,

230 Appleton Place, Peabody Box 74,

Nashville, TN 37203, USA

e-mail: sasha.key@vanderbilt.edu

E. M. Dykens

Vanderbilt Kennedy Center for Research on Human Development, Vanderbilt University,

230 Appleton Place, Peabody Box 40,

Nashville, TN 37203, USA

e-mail: elisabeth.dykens@vanderbilt.edu

\section{A. P. F. Key}

Department of Hearing and Speech Sciences,

Vanderbilt University,

1215 21st Ave S.,

Nashville, TN 37232, USA

more advanced stages of cognitive processing, a larger P3b response to global and local targets was observed in the typical group but not in persons with WS, who instead demonstrated an enhanced P3a to global targets only. The results indicate that in a perceptual task, adults with WS may experience greater than typical global-to-local interference and not allocate sufficient attentional resources to local information.

Keywords Williams syndrome - Local-global processing · Attention $\cdot$ ERP. P3

Williams syndrome (WS) is a genetic disorder caused by a microdeletion of at least 24 genes on chromosome 7 (Hillier et al. 2003) with an estimated prevalence rate of 1:7,500 (Strømme et al. 2002). WS involves mild to moderate levels of intellectual disabilities (Bellugi et al. 2000; Mervis and Klein-Tasman 2000; see Martens et al. 2008 for a review) with pronounced deficits in visuo-spatial processing and construction abilities (Bellugi et al. 1999, 2000; Mervis et al. 2000; Karmiloff-Smith et al. 2003).

Persons with WS often appear unable to draw a complete picture, and instead produce multiple elements without connecting them together (Wang et al. 1995). They also do poorly on tests requiring copying and construction, such as block design tests (Wang et al. 1995; Bellugi et al. 1988, 2000). These observations led some to suggest that persons with WS may be unusual in their perceptual focus on local (i.e., parts) rather than global (i.e., whole) characteristics of visual stimuli (Bihrle et al. 1989; Bellugi et al. 1994, 1999; Deruelle et al. 1999). However, in a Banks-Prinzmetal visual search task, adults with WS appeared to benefit from global information, as the configuration rather than the number of the distracters had a greater effect on performance (Pani et al. 1999). Persons with WS are successful at face recognition (Tager-Flusberg et al. 2003), a skill that is assumed to involve processing of global information, and 
no local-global differences were reported in their drawings of a person (Dykens et al. 2001). Developmental studies in persons with WS demonstrated improvement in the quality of drawing with age, suggesting delayed rather than atypical global processing (Bertrand and Mervis 1996; Bertrand et al. 1997). Observations of improved performance on a block design task when the target pattern was presented in a segmented way (e.g., with slight spaces separating the design elements) also suggested that persons with WS might experience difficulty disengaging from global information (Farran et al. 2001; Mervis et al. 1999).

This inconsistency in conclusions about the local/global processing preferences in WS led Farran and Jarrold (2003) to propose that observed differences in performance may be due to task demands rather than a perceptual bias. Most of the standardized behavioral tests of visuo-spatial skills require more than just visual processing in order to complete the task successfully (Hoffman et al. 2003), including motor ability, memory, planning, and attention. Farran and Jarrold (2003) further suggest that the reported local focus in persons with WS is associated only with construction tasks. Conversely, in a recent study of visuo-spatial performance in persons with WS, Porter and Coltheart (2006) argued that the local bias is attention-based and may be independent of the construction difficulties. They propose at least two subgroups in WS: those with deficits either in perceptual integration or in construction. Other studies have reported high rates of inattention and distractibility in persons with WS (e.g., Pober and Dykens 1996; Greer et al. 1997), and among children, high rates of ADHD, which could complicate behavioral assessments.

Evidence of brain structure or function alterations in WS may shed some light on the visual-spatial deficits in this syndrome (see Jackowski et al. 2009 for a review). Reported structural abnormalities include reduced gray matter density in posterior thalamus and parieto-occipital cortex (MeyerLindenberg et al. 2004; Reiss et al. 2004), the same cortical areas that demonstrate decreased activation during visuospatial tasks (Meyer-Lindenberg et al. 2004; Mobbs et al. 2007). Functionally, these areas belong to the dorsal visual stream that supports spatial processing (Pellicano et al. 2005), including global motion processing (Britten et al. 1992), a reported weakness in WS (Atkinson et al. 2003). Interestingly, the alterations in the dorsal system are particularly noticeable in the left hemisphere (V1, layer 4Ca; Galaburda et al. 2002; see also Boddaert et al. 2006), which is thought to support local processing (Proverbio et al. 1998).

Nevertheless, deficits of the dorsal stream function do not necessarily lead to a lack of ability to process all global information. For example, global form processing can be carried out by the ventral system (Atkinson et al. 1997), which is less affected in WS (Meyer-Lindenberg et al. 2004; Galaburda and Bellugi 2000). However, while in a typical visual system, the dorsal and ventral streams share many connections (Galaburda et al. 2002), the interactions between the dorsal and ventral stream in WS are not entirely typical. Recent fMRI findings identified greater than expected connectivity between the parahippocampal gyrus and other ventral stream structures within the temporal lobe, including superior temporal gyrus (Sarpal et al. 2008), which may explain preserved biological motion processing in WS (Jordan et al. 2002; Reiss et al. 2005). At the same time, there was reduced connectivity between the inferior parietal sulcus (dorsal) and the parahippocampal gyrus (ventral) structures (Sarpal et al. 2008; Epstein et al. 1999), a connection that would typically be responsible for topographic representation of space.

The inconsistency of behavioral findings across tasks and the evidence of alterations in the visual system structures that could impact both local and global processing in WS indicate the need for a more detailed examination of the perceptual and attentional processes associated with processing of hierarchical information in this group. Psychophysiological methods such as event-related potentials (ERPs) may be particularly helpful as they offer the temporal resolution needed to track sensory, perceptual, and attentional processes as they occur in the brain and therefore can complement the existing behavioral and imaging data. ERPs are a portion of electroencephalogram time-locked to a stimulus event (e.g., presentation of a visual display), and reflect brain activity associated with both early sensory and later more advanced cognitive processes elicited by such events (Hugdahl 1995). Specifically, examination of the early peaks occurring within the first 200 ms after stimulus onset (e.g., occipital P1 and N1) would yield information regarding the early sensory and perceptual analysis. Later peaks, such as the frontal P3a and centro-parietal P3b would be more informative about involuntary and voluntary attentional processes, respectively (see Key et al. 2005 for a review).

Behavioral studies of the perceptual time course in typical populations suggest that global information is usually processed first, followed by the more finegrained local analysis, as reflected in faster reaction times for global than local stimuli and the evidence of global-to-local interference in the absence of local-toglobal effects (Navon 1977). This global precedence phenomenon is involuntary (Navon 1991) and can be reduced or eliminated by manipulating perceptual characteristics of the stimuli such as number, size, or spatial arrangement of local elements as well as the overall visual angle and the retinal location (Martin 1979; Ward 1982; Lamb and Robertson 1988). However, sensitivity to stimulus factors does not rule out the possibility that global advantage may have an attentional source as well (Navon and Norman 1983; Ward 1982). 
Findings from prior ERP studies with typical populations show a similar timeline with faster global processing as reflected in shorter latencies of N1, N250, and P3 peaks (Proverbio et al. 1998; Han et al. 1997) possibly due to the low spatial frequencies (i.e., general outline) being available before high frequencies (i.e., details; Hughes et al. 1996). ERP data further revealed that local processing required greater perceptual resources as suggested by a larger posterior P1 (Han et al. 1997, 1999) and more negative N250 (Heinze et al. 1998; Han et al. 1997), particularly over the left hemisphere (Han et al. 1999), thought to specialize in local processing (Proverbio et al. 1998). Interestingly, occipital P1 was larger for local stimuli regardless of the stimulus type (letter vs. object) or visual field position (central vs. peripheral), suggesting that it reflected general selective attention (Han et al. 1997). Global-to-local interference resulted in a reduced N115 for local stimuli when the global characteristics were incongruent (Proverbio et al. 1998), as well as a more negative N2 and less positive P3 (Han et al. 1997). Han et al. (1999) also noted that the frontal lobe is critical for local processing as local-global mismatch increased anterior N2 for local stimuli while decreasing it for global trials. ERPs further demonstrate that local/global processing may be altered by task instructions. Heinze et al. (1998) observed no P1 effects and increased right hemisphere N2 response for global targets in the divided attention condition (attend to both levels) but no effect in the selective attention condition when only one level was attended to.

To date, no comparable ERP studies have been conducted in persons with WS. The only ERP study relevant to the ability to process the whole stimulus in addition to its local parts examined automatic perceptual completion (Kanizsa square illusions) and reported no differences in N1 response for the illusory and no contour stimuli in persons with WS, even though behavioral evidence indicated that they indeed experienced the illusion, suggesting altered or possibly delayed early perceptual processing in WS (Grice et al. 2003).

The purpose of the current study was to examine the preferred level of perceptual analysis in persons with WS and the role of attention in the processing of hierarchical stimuli. We chose the letter stimuli similar to the ones originally developed by Navon (1977) because such stimuli have been used before in copying tasks to demonstrate a local bias in WS and also because letter identification should be a nearly automatic process in adults. The stimuli were presented in an oddball paradigm with the general instructions to look for the target letter but no explicit requirement to direct attention toward the global or local level (i.e., an implicit divided attention task; Roalf et al. 2006). This task design provided an opportunity to assess the preferred level of perceptual analysis for each individual rather than the ability to process global or local information following an external demand. We hypothesized that targets presented at the preferred level of processing would be detected faster and more accurately. Furthermore, if differences in preferred level of analysis were due to early perceptual processing (as proposed by Grice et al. 2003), preferred target level effects would be reflected in the amplitude of $\mathrm{P} 1$ and/or N1 responses. Finally, if any perceptual bias in WS is attention-based, the target letter presented at the preferred level should elicit a larger P3b response than non-preferred level targets. However, if difficulties in tasks requiring local/global processing are due to general inability to maintain attention to complex stimuli, we expected a more pronounced anterior P3a (reflecting involuntary orienting to rare/novel stimuli) and reduced or absent centro-parietal $\mathrm{P} 3 \mathrm{~b}$ for both local and global targets.

\section{Method}

\section{Participants}

Twenty-one young adults with WS (12 males; $\mathrm{M}$ age=26.24, $\mathrm{SD}=8.26$ years) participated in the study. Five participants were left-handed, the rest were right-handed $(\mathrm{M} \mathrm{LQ}=0.478$, $\mathrm{SD}=0.8$ ) as determined by Edinburgh Handedness Inventory (Oldfield 1971). IQ was assessed by the Kaufman Brief Intelligence Test-2 (K-BIT-2; Kaufman and Kaufman 2004), which was individually administered by trained research assistants. The mean total IQ for the WS group was 74.25 $(\mathrm{SD}=17.19)$, with better performance in verbal versus nonverbal domains, $\mathrm{M}=79.55, \mathrm{SD}=13.75$ and $\mathrm{M}=69.20$, $\mathrm{SD}=24.78$, respectively $(p=0.02)$.

A group of 16 typical participants (seven males; $\mathrm{M}$ age $=$ 29.64, $\mathrm{SD}=11.77$ years) was included to document typical task performance. One participant was left-handed, the rest were right-handed $(\mathrm{M} L Q=0.716, \mathrm{SD}=0.34)$. Typical controls were university students, or college graduates employed in the community, with presumed adequate cognitive and adaptive skills.

All participants (and their parents/guardians when needed) provided written informed consent, and the study was conducted with approval from the Institutional Review Board of Vanderbilt University, in accordance with the Helsinki Declaration of 1975, as revised in 2000 (World Medical Association Declaration of Helsinki 2000). All participants reported normal or corrected-to-normal vision.

\section{Stimuli}

Capital letters "S", " $\mathrm{H}$ ", and " $\mathrm{O}$ " served as the stimuli and were presented in black against the white background. Each letter 
could appear as a global form (big letter) or a local element (small letters) but never in the same trial (i.e., global and local characteristics were always incongruent, such as a big " $\mathrm{H}$ " made of little "S"). Global stimuli were $6 \mathrm{~cm}$ high and $3.5 \mathrm{~cm}$ wide. Local elements were $0.75 \mathrm{~cm}$ high and $0.5 \mathrm{~cm}$ wide. From the viewing distance of $90 \mathrm{~cm}$, the stimuli subtended respective visual angles of $3.8^{\circ} / 0.48^{\circ}(\mathrm{h}) \times 2.2^{\circ} / 0.32^{\circ}(\mathrm{w})$.

\section{Electrodes}

A high-density array of $128 \mathrm{Ag} / \mathrm{AgCl}$ electrodes embedded in soft sponges (Geodesic Sensor Net, EGI, Inc., Eugene, OR, USA) was used to record the ERPs. Electrode impedance levels were at or below $40 \mathrm{kOhm}$ as checked before and after testing. During data collection, data were sampled at $250 \mathrm{~Hz}$ with the filters set to $0.1-30 \mathrm{~Hz}$. All electrodes were referred to $\mathrm{Cz}$ (vertex) and then re-referenced offline during data analysis to an average reference.

\section{Procedure}

The stimuli were presented in an oddball paradigm. Participants were asked to look for letter " $\mathrm{H}$ ", and indicate their response by pressing one of the two buttons (yes/no). The target letter could appear at the local or global level with equal probability ( $20 \%$ each). To avoid biasing participants' attention to a particular level of perceptual analysis, no specific instructions were given regarding the local or global elements. To ensure participants' comprehension of the task, instructions included examples of other hierarchical letters and verbal descriptions identifying both global and local features (e.g., "Here's a 'T' made of 'U's").

Each trial began with a $500 \mathrm{~ms}$ fixation point followed by a $250 \mathrm{~ms}$ presentation of the stimulus letter. The response collection window included up to $2,000 \mathrm{~ms}$. Intertrial interval varied randomly between 1,800 and 2,800 ms to prevent habituation and development of trial onset expectations. Stimulus presentation was controlled by E-prime (v.1.2, PST, Inc., Pittsburgh, PA, USA). The entire task included 150 trials (90 distracters, 30 local targets, 30 global targets). On average, task duration was approximately $10 \mathrm{~min}$. A researcher was present in the room to monitor participants' behavior. During the periods of inattention or motor activity, stimulus presentation was suspended until the participant was ready to continue with the task.

\section{Data analysis}

Behavioral data

Accuracy and reaction time data were collected for each stimulus condition and submitted to separate planned comparisons to examine condition differences (standard vs. local target, standard vs. global target, and global vs. local target).

\section{ERP}

Individual ERPs were derived by segmenting the ongoing EEG to include a $100-\mathrm{ms}$ prestimulus baseline and a $700-$ ms post-stimulus interval. To ensure that all conditions (standard, local target, and global target) had a comparable number of trials, only the standard trials preceding either of the targets were selected for the analysis. Trials contaminated by ocular and movement artifacts were rejected from further analysis using an automated screening algorithm in NetStation $^{1}$ followed by a manual review. Electrodes identified as "bad" (poor signal quality on $20 \%$ or more of the trials) were replaced by reconstructing their data using spherical spline interpolation procedures. If more than $15 \%$ of the electrodes within a trial were deemed bad, the entire trial was discarded. The retention rates were comparable across conditions and groups (controls, standard $=29.75 \pm 8.58$, local $=22.38 \pm 5.23$, and global $=21.94 \pm$ 5.67 and WS, standard $=22.36 \pm 8.56$, local $=18.32 \pm 5.76$, and global $=18.27 \pm 4.96$ ).

Following artifact screening, individual ERPs were averaged and baseline-corrected by subtracting the average microvolt value across the $100-\mathrm{ms}$ prestimulus interval from the post-stimulus segment. To reduce the number of electrodes in the analysis, data from 128 electrodes were submitted to a spatial principle components analysis (sPCA), an objective and replicable statistical approach that identified a small set of 'virtual electrodes' (see Fig. 1), each representing a spatially contiguous group of electrodes with similar ERP waveforms (see Spencer et al. 1999). Specific electrodes comprising each cluster were identified using the criterion of factor loadings of $|0.6|$ or greater.

Next, maximum ${ }^{2}$ amplitude and latency measures for the occipital P1 (70-150 ms) and N150 (150-220 ms) responses as well as centro-parietal P3b (300-600 ms) and frontal P3a (200-400 ms) components were derived using an automated scoring tool in NetStation 4.2. Time windows were selected a priori based on intervals utilized in previously published studies of local-global processing and visual oddball paradigms. The resulting values were averaged across the electrodes within a cluster (see Table 1)

\footnotetext{
${ }^{1}$ The same voltage shift threshold values were applied to all participants.

${ }^{2}$ While the choice of mean vs. peak amplitude measures is often a matter of preference (Handy, 2005) and depends largely on data quality, reanalyzing these data using mean amplitude measures did not result in any changes in the pattern of condition differences (with the exception of the $p$ value for one of the seven reported effects for the typical group increasing to 0.065 ).
} 


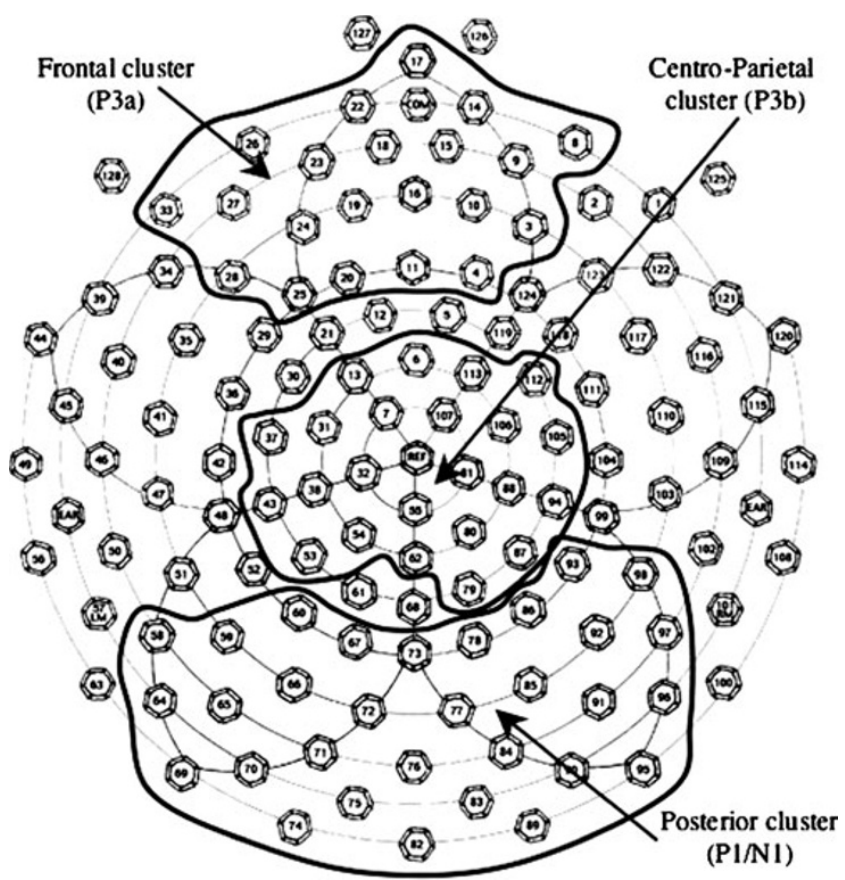

Fig. 1 Electrode clusters identified by spatial PCA and used in the statistical analyses

and entered into separate repeated-measures ANOVAs to examine condition effects followed by planned comparisons (standard vs. local target and standard vs. global target).

\section{Results}

Typical group

Analysis of behavioral performance demonstrated the expected global precedence effect where global targets were associated with shorter reaction times (RT) than standard stimuli (515.35 ms vs. $545.61 \mathrm{~ms}, t(15)=2.164, p=0.047, d=$ $0.54)$ or local targets $(515.35 \mathrm{~ms}$ vs. $539.28 \mathrm{~ms}, t(15)=$ $3.350, p=0.004, d=0.84$; Fig. 2b). There were no RT differences between local targets and standard stimuli ( $t$ $(15)=0.439, p=0.667)$. Accuracy rates for all three conditions were near perfect (see Fig. 2a); however, both global (96.94\%) and local (93.50\%) targets were detected slightly less accurately than standard $(99.13 \%)$ stimuli (global vs. standard, $t(15)=2.108, p=0.052, d=0.53$ and local vs. standard, $t(15)=2.79, p=0.014, d=0.70)$. No other differences in accuracy between conditions reached significance.

Analysis of the ERPs in the early sensory processing range revealed a trend for a condition main effect for $\mathrm{P} 1$ amplitude $(F(2,30)=2.986, p=0.082)$ due to a reduced amplitude for global targets compared to standard stimuli $(t(15)=2.308, p=$ $0.036, d=0.58$ ). A main effect of condition was present for
N150 amplitude $(F(2,30)=7.851, p=0.002)$ where global and local targets both elicited a more negative peak than the standard stimuli $(t(15)=2.842, p=0.012, d=0.71 ; t(15)=$ $3.586, p=0.003, d=0.90$, respectively; Fig. $3 c$ ). There were no latency effects for $\mathrm{P} 1(F<1, p=0.595)$ or $\mathrm{N} 150(F(2,30)=$ 2.312, $p=0.141$ ).

In the later interval associated with more advanced cognitive processing, condition differences were present for the centro-parietal P3b amplitude $(F(2,30)=6.929, p=0.007)$ and latency $(F(2,30)=4.528, p=0.019)$. Both global and local targets were associated with larger (more positive) $\mathrm{P} 3 \mathrm{~b}$ responses compared to the standard stimuli $(4.66 / 4.77 \mu \mathrm{V}$ vs. $3.44 \mu \mathrm{V}, t(15)=3.265, p=0.005, d=0.82$ and $t(15)=$ $2.680, p=0.017, d=0.67$, respectively) but the $\mathrm{P} 3 \mathrm{~b}$ response for the local targets was delayed compared to the standard stimuli (450 vs. $421 \mathrm{~ms}, t(15)=2.682, p=0.017, d=0.67$; Fig. 3b).

Table 1 Amplitude and latency values of the three selected peaks for participants with WS and typical development

\begin{tabular}{|c|c|c|c|c|c|}
\hline \multirow[t]{2}{*}{ Occipital P1 } & \multirow[t]{2}{*}{ Condition } & \multicolumn{2}{|c|}{ WS group } & \multicolumn{2}{|c|}{ TD group } \\
\hline & & M & SD & M & SD \\
\hline \multirow[t]{3}{*}{ Amplitude $(\mu \mathrm{V})$} & Global & 0.97 & 2.54 & 0.15 & 1.77 \\
\hline & Local & 1.82 & 3.24 & 0.74 & 1.96 \\
\hline & Standard & 2.37 & 2.63 & 1.07 & 1.31 \\
\hline \multirow[t]{3}{*}{ Latency (ms) } & Global & 111.52 & 20.71 & 104.16 & 17.98 \\
\hline & Local & 120.73 & 19.58 & 106.37 & 16.53 \\
\hline & Standard & 120.93 & 17.78 & 107.99 & 13.72 \\
\hline \multicolumn{6}{|l|}{ Occipital N150 } \\
\hline \multirow[t]{3}{*}{ Amplitude $(\mu \mathrm{V})$} & Global & -5.03 & 3.54 & -5.95 & 3.28 \\
\hline & Local & -4.50 & 3.88 & -6.16 & 3.02 \\
\hline & Standard & -3.84 & 3.34 & -4.79 & 3.10 \\
\hline \multirow[t]{3}{*}{ Latency (ms) } & Global & 184.88 & 17.05 & 175.96 & 13.64 \\
\hline & Local & 184.25 & 21.13 & 182.19 & 18.32 \\
\hline & Standard & 183.23 & 19.12 & 173.02 & 12.76 \\
\hline \multicolumn{6}{|l|}{ Centro-parietal P3b } \\
\hline \multirow[t]{3}{*}{ Amplitude $(\mu \mathrm{V})$} & Global & 4.87 & 2.56 & 4.66 & 3.19 \\
\hline & Local & 4.74 & 2.20 & 4.77 & 3.85 \\
\hline & Standard & 4.21 & 1.63 & 3.44 & 2.77 \\
\hline \multirow[t]{3}{*}{ Latency (ms) } & Global & 413.91 & 60.99 & 429.30 & 62.95 \\
\hline & Local & 426.43 & 68.36 & 450.78 & 48.80 \\
\hline & Standard & 416.15 & 59.29 & 421.21 & 53.68 \\
\hline \multicolumn{6}{|l|}{ Frontal P3a } \\
\hline \multirow[t]{3}{*}{ Amplitude $(\mu \mathrm{V})$} & Global & 7.22 & 4.43 & 6.56 & 2.47 \\
\hline & Local & 5.41 & 3.79 & 6.08 & 2.81 \\
\hline & Standard & 5.19 & 3.15 & 4.81 & 2.21 \\
\hline \multirow[t]{3}{*}{ Latency (ms) } & Global & 298.17 & 55.59 & 247.07 & 42.38 \\
\hline & Local & 310.91 & 51.19 & 255.07 & 50.92 \\
\hline & Standard & 324.09 & 48.45 & 258.44 & 56.48 \\
\hline
\end{tabular}

$M$ mean, $S D$ standard deviation 
a
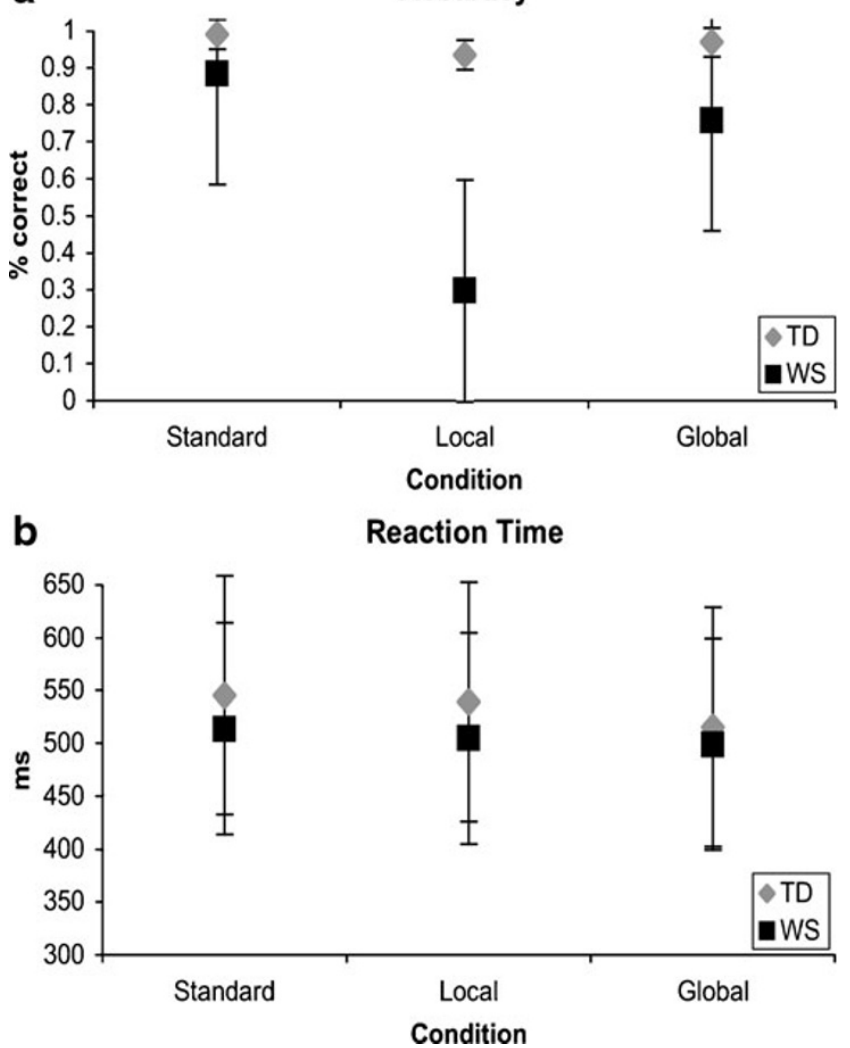

Fig. 2 Average behavioral accuracy a and reaction times $\mathbf{b}$ for each condition. Error bars standard error

Condition differences were also observed for the frontal P3a amplitude $(F(2,30)=5.193, p=0.017)$ thought to reflect involuntary orienting to rare stimuli. Both global and local targets elicited more positive responses compared to the standard stimuli $(6.56 / 6.07 \mu \mathrm{V}$ vs. $4.81 \mu \mathrm{V}, t(15)=4.186, p=$ $0.001, d=1.05$ and $t(15)=2.327, p=0.034, d=0.58$, respectively; Fig. 3a). There were no condition effects for P3a latency $(F<1, p=0.529)$.

\section{Williams syndrome group}

Analysis of the RT data failed to identify any differences among the conditions $(t \leq 1, p>0.30)$. In the accuracy domain, both global $(75.10 \%)$ and local $(31.14 \%)$ targets were detected less accurately than the standard $(88.05 \%)$ stimulus (standard vs. global, $t(20)=2.861, p=0.010$, $d=0.62$ and standard vs. local, $t(20)=5.935, p<0.0001$, $d=1.30$ ). Persons with WS detected global targets more accurately than local targets $(t(20)=4.397, p<0.0001$, $d=0.96$; see Fig. 2a).

In the early processing period, there was a condition effect for P1 amplitude $(F(2,40)=6.490, p=0.006)$ and latency $(F(2,40)=4.293, p=0.035)$. Post-hoc analyses indicated that relative to the standard stimuli, global targets elicited a smaller P1 peak $(t(20)=4.362, p<0.001, d=0.95)$ with shorter latency $(t(20)=2.298, p=0.033, d=0.50)$. There was also a condition main effect for the amplitude of the N150 response $(F(2,40)=4.690, p=.015$; Fig. 3c) where global targets were associated with more negative amplitudes than the standard stimuli $(t(20)=2.929, p=$ $0.008, d=0.64)$. No latency effects $(F<1, p=0.799)$ were present for N150.

There were no significant condition effects in the later processing period for the centro-parietal $\mathrm{P} 3 \mathrm{~b}$ amplitude $(F(2,40)=1.329, p=0.276)$ or latency $(F<1, p=0.522)$. However, the frontal $\mathrm{P} 3 \mathrm{a}$ was characterized by the main effect of condition for amplitude $(F(2,40)=6.259, p=0.008)$ and latency $(F(2,40)=3.966, p=0.027)$. Compared to the standard stimuli, global targets elicited more positive ERPs (7.22 vs. $5.18 \mu \mathrm{V} ; t(20)=3.012, p=0.007, d=0.66$; Fig. $3 \mathrm{a}$ ) with shorter latencies (298 vs $324 \mathrm{~ms} ; t(20)=2.448, p=$ $0.024, d=0.53$ ). None of the comparisons involving local targets reached significance.

\section{Discussion}

This study was designed to examine the preferred level of perceptual analysis in persons with WS and to evaluate contributions of the attentional processes. To exclude potential confounding influences of other abilities typically needed for successful performance of visuo-spatial tasks with hierarchical stimuli, we utilized a purely perceptual task that did not involve any motor (e.g., copying) or construction components. The behavioral results indicated that in the absence of specific instructions directing attention to a particular level of a hierarchical stimulus (i.e., using an implicit divided attention task), persons with WS are subject to the global precedence effect previously observed in typical populations as reflected by higher accuracy in detection of global than local targets. The absence of the reaction time shortening for global targets that was observed in the typical comparison group could be attributed to greater heterogeneity of the WS sample as the trend to shorter RTs in response to global targets was present.

Examination of the psychophysiological measures revealed potential mechanisms underlying behavioral similarities and differences between the two groups. During the early perceptual processing, persons with WS and typical participants demonstrated a similar modulation of the occipital P1/N1 responses to global compared to the standard stimuli. Traditionally, P1 amplitude is thought to reflect encoding of form in the ventral "what" pathway (Mangun et al. 1993) and has been shown to vary based on the number of objects for identification (one at the global level, multiple at the local level; Han et al. 1999, 2000). Prior studies of hierarchical processing utilizing selective 
Fig. 3 ERP waveforms for the three stimulus conditions recorded over frontal a, centroparietal b, and occipital c scalp areas in typical controls (left column) and persons with WS (right column)
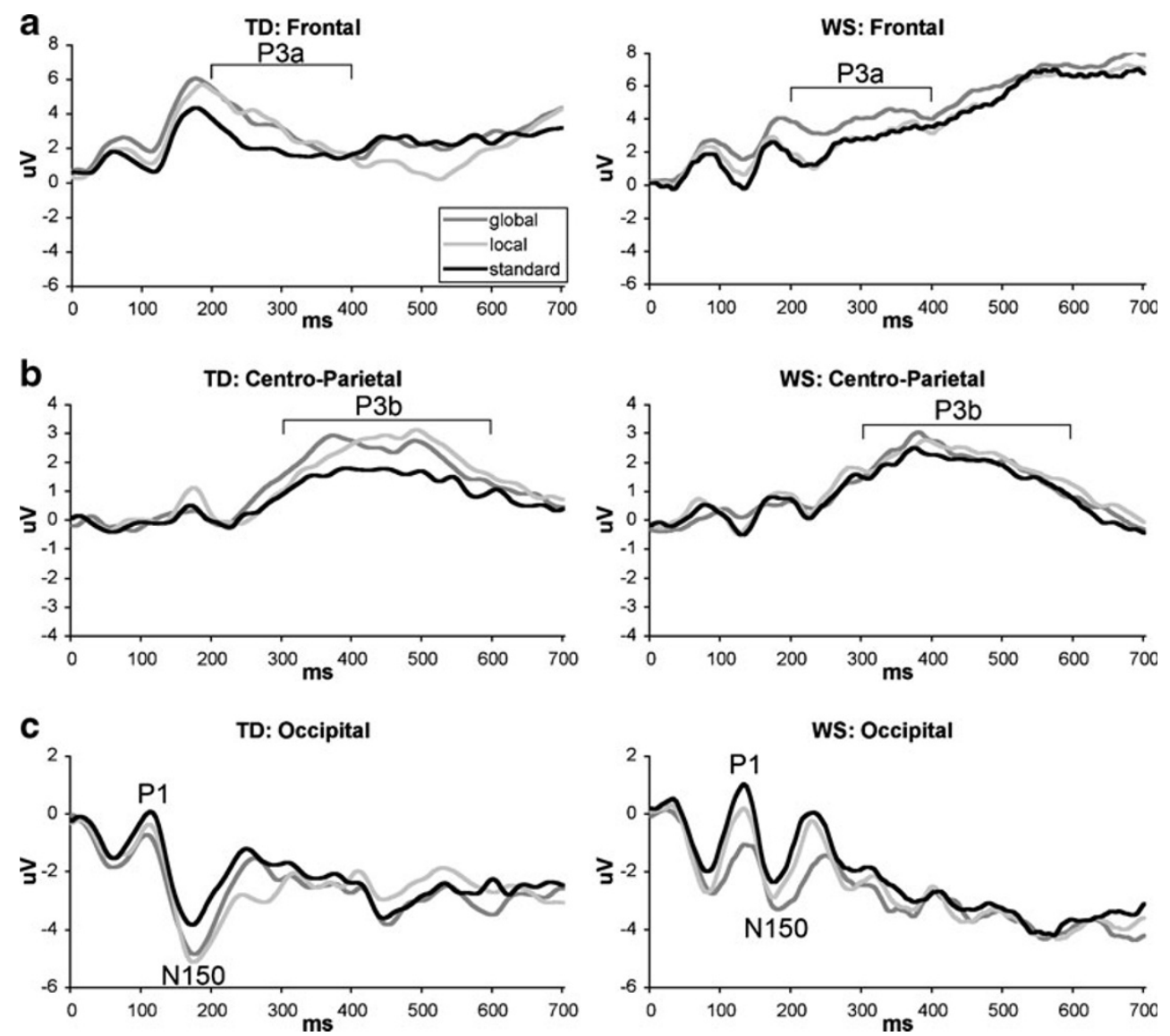

attention tasks frequently reported a smaller P1 for stimuli processed at global level (e.g., Han et al. 1997, 1999; Boeschoten et al. 2005). Reduced P1 amplitudes for global targets relative to the standard stimuli in the present study suggest that persons with WS and typical adults detected the overall stimulus configuration at the early stage of perceptual analysis. The standard stimuli were associated with larger P1 responses because they could be functionally equivalent to the local stimuli in the previous studies as both required processing of the local features before a decision about the presence or absence of the target letter could be made.

The global target stimuli were also associated with an enhanced N150 in both groups, further underscoring between-group similarities in early processing. This peak reflects ability to attend to basic stimulus characteristics (Vogel and Luck 2000), including spatial properties of the stimuli (Mangun et al. 1993). A similar increase in N150 amplitude was observed for the local targets in the typical comparison group, indicative of their ability to direct attention to either level of the hierarchical stimuli. The presence of the increased N150 to local stimuli in the absence of a P1 effect in the typical sample may be consistent with the time line of early perceptual analysis where local processing requires a separate step of reorienting attention away from the global level (which is usually attended to first) as proposed in a behavioral study by Stoffer (1993). In contrast, in the WS group, amplitudes of N150 to the local targets did not differ from the standard stimuli. In line with the interpretation that the increase in amplitude of this peak reflects enhanced processing of the attended location (Coull 1998; Luck 1995), the observed pattern of results suggests that persons with WS successfully attended to the overall spatial configuration of the stimuli, but were less likely to orient to the local information.

The finding of enhanced N150 amplitude for global compared to standard stimulus in persons with WS appears inconsistent with the data reported by Grice et al. (2003) who observed no modulation of this response to stimuli where local elements created an illusion of a contour outlining a global figure (Kanizsa square) compared to the same local stimuli arranged in a pattern that did not produce the illusion. However, inconsistencies across studies are likely due to differences in tasks. Grice et al. (2003) used a passive paradigm where participants were asked to pay attention to faces rather than the Kanizsa stimuli. Persons with WS are often characterized as hypersociable (Jones et 
al. 2000) and faces are of keen interest to them (Bellugi et al. 2000; Laing et al. 2002; Mervis and Bertrand 1997). Thus, the Kanizsa stimuli could actually be treated as distracters, and therefore not receive much attention from the participants, leading to the observed reduction in the N150 amplitude, even though behavioral evidence suggested that they indeed experienced the Kanizsa illusion.

The lack of N150 enhancement for the local targets in persons with WS could be attributed to poor visual acuity that is not uncommon in this syndrome (e.g., Atkinson et al. 2001) as poor vision could bias a person to be better at processing low spatial frequencies (i.e., global stimulus characteristics). ${ }^{3}$ However, in the study by Atkinson et al. (2001), no correlation was observed between visual deficits and performance on visuo-spatial tasks. Furthermore, all participants in the present sample reported normal or corrected-to-normal vision. Also, incidentally, the local stimuli in the current study corresponded in size to the letters on a 20/20 line of the Snellen chart but were viewed from a distance of three rather than $20 \mathrm{ft}$. Thus, we are reasonably confident that all participants were able to clearly see both local and global features of the stimuli and the observed differences in ERPs are reflecting differences in the preferred mode of perceptual analysis.

Analysis of ERP at the more advanced stages of visual processing involving voluntary attention revealed more pronounced differences in hierarchical processing of persons with WS. The typical participants demonstrated the expected increase in the centro-parietal P3b amplitude for both local and global targets, with the local targets eliciting a delayed response reflecting increased duration of stimulus evaluation (Donchin and Coles 1988) that was also reflected in their delayed RT values. In sharp contrast to controls, the centro-parietal P3b effect was absent in the participants with WS.

One interpretation would be that persons with WS did not understand the task or had greater difficulty maintaining attention to the task and therefore did not detect the targets. Clinical evidence suggests that persons with WS do often experience attentional problems (Greer et al. 1997). General inattention, however, may not be the most optimal explanation for the observed pattern of results as behavioral accuracy for detection of global targets and identification of no-target distracters (standard stimuli) was greater than chance. Comparison of the P3b amplitudes in persons with WS to those of the typical group suggests that the lack of target-related differences could be due to the increased P3b amplitude to the standard stimuli rather than the reduction of the $\mathrm{P} 3 \mathrm{~b}$ response to targets, as the latter amplitudes were comparable in the two groups. We attribute these results to

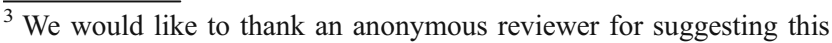
possibility.
}

the potentially challenging nature of the task-because targets and distracters each had more than one physical form and were presented relatively briefly, the participants with WS might have engaged into overly extensive processing of every hierarchical stimulus to ensure that they responded correctly. In other words, the reasons underlying observed lack of condition differences in $\mathrm{P} 3 \mathrm{~b}$ amplitude were not exclusively perceptual (letter recognition relies on stable memory traces and is a nearly automatic process in adults) but reflected over-engaged attention to the frequent distracter stimuli and insufficient ability to develop a mind set for the distracters vs. targets. This interpretation is consistent with the nature of the $\mathrm{P} 3 \mathrm{~b}$ response, which is thought to reflect the amount of processing resources available (Donchin et al. 1986), the quality of selection (Johnstone et al. 1996), and attention allocation (Jonkman et al. 2000).

It is also possible that the lack of the observed posterior $\mathrm{P} 3 \mathrm{~b}$ effect was due to heterogeneity in perceptual processing skills in WS, as previously reported by Porter and Coltheart (2006). Indeed, in a small subset of participants with WS who demonstrated a local bias (i.e., higher accuracy of detecting local than global targets), there was a centro-parietal P3b effect for the local targets. Regrettably, the very small number of such participants in the current sample $(n=3)$ did not allow for any meaningful statistical analyses and further studies should examine this possibility in greater detail.

An alternative interpretation could attribute the observed lack of P3b amplitude modulation by the frequent distracters and/or rare target stimuli to the general cognitive impairment in persons with WS. Although participants with WS performed significantly worse than the typical comparison group, we feel that their overall relatively high behavioral performance ( $>75 \%$ accuracy for two of the three conditions) as well as similarities in ERP amplitudes to target stimuli across the two participant groups argue against this possibility. Nevertheless, inclusion of an IQ-matched comparison group in follow-up studies would allow for more optimal acrossgroup comparisons and therefore help clarify the roles of general cognitive functioning and genetic characteristics in hierarchical perceptual processing.

Finally, the lack of the P3b effects may be due to the atypical brain morphology in WS. Although brain sources of the $\mathrm{P} 3 \mathrm{~b}$ are not clearly identified, at least some are expected to be located in the parahippocampal gyrus or thalamus (Katayama et al. 1985) and/or deep within the temporoparietal area or in the temporoparietal junction (Knight et al. 1988), areas identified as atypical in structure or connectivity in WS (Sarpal et al. 2008; Epstein et al. 1999).

Both participant groups demonstrated involuntary orienting to rare target stimuli as reflected by the frontal P3a response. However, in the WS group, the effect was 
again limited to the global targets, confirming that persons with WS processed the global configuration of the hierarchical stimuli and did so faster compared to standard stimuli (which would require processing of local elements before a response could be selected) as evidenced by shorter P3a latency. The lack of such orienting response to local targets in persons with WS further suggests altered ability to attend to or otherwise notice local details when attention is not explicitly directed toward them.

Taken together, these findings indicate that when presented with a hierarchical stimulus, persons with WS tend to process global configuration first (similar to typical controls) but demonstrate potential impairment in the ability to process local details. Hughes et al. (1996) suggested that global and local information are processed in parallel but at different rates, with global information becoming available sooner. This would be advantageous from the evolutionary perspective as global information may be more helpful for quickly determining the overall identity of objects (Navon 1991) and may facilitate analysis and integration of perceptual elements (Marr 1982; Neisser 1967). However, global information may also interfere with the processing of details (see Kimchi 1992 for a review). ERP and behavioral evidence in our sample suggest that this may indeed be the case in persons with WS. In the absence of an explicit requirement to attend to it, local information processing in persons with WS may not be initiated or be atypically delayed to the point that the process does not get completed.

This idea appears to be at odds with the behavioral studies utilizing the same type of stimuli in copying tasks and demonstrating a local bias in WS. However, in line with the conclusion of Farran and Jarrold (2003), it is important to remember the differences in specific task requirements. When presented with a copying task or any other drawing, one has to start with an element and proceed to create the whole image piece-by-piece. Our ERP evidence suggests that attention to local features in WS may not happen automatically and therefore could require substantial attentional resources, potentially leaving nothing for the construction part of the task (i.e., analyzing and replicating the spatial layout of the elements).

In sum, our results suggest that reported visuo-spatial difficulties in persons with WS may be due, at least in part, to the atypical brain mechanisms underlying attention to local information during early perceptual analysis as well as at the more advanced stages of cognitive processing. This alteration may manifest at the behavioral level as a greater than typical global-to-local interference (as evidenced by improved performance on block design when the target image is segregated into individual blocks; Farran et al. 2001; Mervis et al. 1999), as well as by difficulty in establishing effective memory traces for frequently experi- enced visual inputs (e.g., difficulty utilizing navigational landmarks in a familiar area, Nardini et al. 2008). Consequently, tasks requiring processing of the local features of a complex visual stimulus may demand increased attentional resources, thus limiting their availability for other aspects of performance (e.g., error monitoring). Future studies are needed to determine if difficulties attending to local information relate to inefficient executive functioning, including the inability to effectively select the level of visual analysis needed for successful task performance.

Acknowledgments This research was supported by National Institute of Child Health and Development Grant P30HD15052 to the Vanderbilt Kennedy Center for Research on Human Development, and by a Vanderbilt University Discovery grant (EMD).

We would like to thank the participants and their families for their support of the study. We are grateful to Elizabeth Roof for assistance in coordinating research participants and to Susan M. Williams and Aikaterini Ntourou for their help with acquisition and processing of the ERP data.

Conflict of interest The authors declare no conflicts of interest.

\section{References}

Atkinson J, King JA, Braddick OJ, Nokes L, Anker S, Braddick F. A specific deficit of dorsal stream function in Williams' syndrome. NeuroReport. 1997;8:1919-22.

Atkinson J, Anker S, Braddick O, Nokes L, Mason A, Braddick F. Visual and visuospatial development in young children with Williams syndrome. Dev Med Child Neurol. 2001;43:330-7.

Atkinson J, Braddick O, Anker S, Curran W, Andrew R, Wattam-Bell $\mathrm{J}$, et al. Neurobiological models of visuospatial cognition in children with Williams syndrome: measures of dorsal-stream and frontal function. Dev Neuropsychol. 2003;23(12):139-72.

Bellugi U, Sabo H, Vaid J. Spatial deficits in children with Williams syndrome. In: Stiles-Davis J, Kritchevsky M, Bellugi U, editors. Spatial cognition: brain bases and development. Hillsdale: Erlbaum Press; 1988. p. 273-98.

Bellugi U, Wang P, Jernigan TL. Williams syndrome: an unusual neuropsychological profile. In: Broman S, Grafman J, editors. Atypical cognitive deficits in developmental disorders: implications for brain function. Hillsdale: Lawrence Erlbaum; 1994. p. $23-56$.

Bellugi U, Lichtenberger L, Mills D, Galaburda A, Korenberg JR. Bridging cognition, the brain and molecular genetics: evidence from Williams syndrome. Trends Neurosci. 1999;22:197-207.

Bellugi U, Lichtenberger L, Jones W, Lai Z, St. George M. The neurocognitive profile of Williams syndrome: a complex pattern of strengths and weaknesses. J Cogn Neurosci. 2000;12 Suppl $1: 7-29$.

Bertrand J, Mervis C. Longitudinal analysis of drawings by children with Williams syndrome: preliminary results. Vis Arts Res. 1996;22:19-34.

Bertrand J, Mervis C, Eisenberg J. Drawing by children with Williams syndrome: a developmental perspective. Dev Neuropsychol. 1997; 13:41-67.

Bihrle A, Bellugi U, Delis D, Marks S. Seeing either the forest or the trees: dissociation in visuospatial processing. Brain Cogn. 1989;11:37-49. 
Boddaert N, Mochel F, Meresse I, Seidenwurm D, Cachia A, Brunelle F, et al. Parieto-occipital grey matter abnormalities in children with Williams syndrome. Neuroimage. 2006;30(3):721-5.

Boeschoten M, Kemner C, Kenemans J, van Engeland H. The relationship between local and global processing and the processing of high and low spatial frequencies studied by event-related potentials and source modeling. Cogn Brain Res. 2005;24:228-36.

Britten KH, Shadlen MN, Newsome WT, Movshon JA. The analysis of visual motion: a comparison of neuronal and psychophysical performance. J Neurosci. 1992;12:4745-65.

Coull J. Neural correlates of attention and arousal: insights from electrophysiology, functional neuroimaging and psychopharmacology. Prog Neurol. 1998;55:343-61.

Deruelle C, Mancini J, Livet M, Casse-Perrot C, de Schonen S. Configural and local processing of faces in children with Williams syndrome. Brain Cogn. 1999;41:276-98.

Donchin E, Coles M. Is the P300 component a manifestation of context updating? Behav Brain Sci. 1988;11:357-427.

Donchin E, Miller GA, Farwell LA. The endogenous components of the event-related potential - a diagnostic tool? Prog Brain Res. 1986;70:87-102.

Dykens E, Rosner B, Ly T. Drawings by individuals with Williams syndrome: are people different from shapes? Am J Ment Retard. 2001;106(1):94-107.

Epstein R, Harris A, Stanley D, Kanwisher N. The parahippocampal place area: recognition, navigation, or encoding? Neuron. 1999;23(1):115-25.

Farran EK, Jarrold C. Visuospatial cognition in Williams syndrome: reviewing and accounting for the strengths and weaknesses in performance. Dev Neuropsychol. 2003;23(1-2):173-200.

Farran EK, Jarrold C, Gathercole SE. Block design performance in the Williams syndrome phenotype: a problem with mental imagery? J Child Psychol Psychiatry. 2001;42(6):719-28.

Galaburda AM, Bellugi U. V. Multi-level analysis of cortical neuroanatomy in Williams syndrome. J Cogn Neurosci. 2000;12 Suppl 1:74-88.

Galaburda AM, Holinger DP, Bellugi U, Sherman GF. Williams syndrome: neuronal size and neuronal-packing density in primary visual cortex. Arch Neurol. 2002;59(9):1461-7.

Greer M, Brown F, Pai S, Choudry S, Klein A. Cognitive, adaptive, and behavioral characteristics of Williams syndrome. Am J Med Genet. 1997;74:521-5.

Grice S, de Haan M, Halit H, Johnson M, Csibra G, Grant J, et al. ERP abnormalities of illusory contour perception in Williams syndrome. NeuroReport. 2003;14(14):1773-7.

Han S, Fan S, Chen L, Zhuo Y. On the different processing of wholes and parts: a psychophysiological analysis. J Cogn Neurosci. 1997;9(5):687-98.

Han S, Fan S, Chen L, Zhuo Y. Modulation of brain activities by hierarchical processing: a high-density ERP study. Brain Topogr. 1999;11(3):171-83.

Han S, He, X, Woods D. Hierarchical processing and level-repetition effect as indexed by early brain potentials. Psychophysiology, 2000;37:817-30.

Handy TC. Basic principles of ERP quantification. In: Handy TC, editor. Event-related potentials: A methods handbook Cambridge: The MIT Press, 2005, pp. 33-55.

Heinze H, Hinrichs H, Scholz M, Burchert W, Mangun G. Neural mechanisms of global and local processing: a combined PET and ERP study. J Cogn Neurosci. 1998;10:485-98.

Hillier LW, Fulton RS, Fulton LA, Graves TA, Pepin KH, WagnerMcPherson C, et al. The DNA sequence of chromosome 7. Nature. 2003;424:157-64

Hoffman J, Landau B, Pagani B. Spatial breakdown in spatial construction: evidence from eye fixations in children with Williams syndrome. Cogn Psychol. 2003;46(3):260-301.
Hugdahl K. Psychophysiology: the mind-body perspective. Cambridge: Harvard University Press; 1995.

Hughes HC, Nozawa G, Kitterle F. Global precedence, spatial frequency channels, and the statistics of natural images. J Cogn Neurosci. 1996;8(3):197-230.

Jackowski A, Rando K, de Araújo C, Grego Del Cole C, Silva I, Tavares de Lacerda A. Brain abnormalities in Williams syndrome: a review of structural and functional magnetic resonance imaging findings. Eur J Paediatr Neurol. 2009;13(4):305-16.

Johnstone SJ, Barry RJ, Anderson JW, Coyle SF. Age-related changes in child and adolescent event-related potential component morphology, amplitude and latency to standard and target stimuli in an auditory oddball task. Int J Psychophysiol. 1996;24:223-38.

Jones W, Bellugi U, Lai Z, Chiles M, Reilley J, Lincoln A, et al. Hypersociability in Williams syndrome. J Cogn Neurosci. 2000;12:30-46.

Jonkman LM, Kemner C, et al. Attentional capacity, a probe ERP study: differences between children with attention-deficit hyperactivity disorder and normal control children and effects of methylphenidate. Psychophysiology. 2000;37:334-46.

Jordan H, Reiss JE, Hoffman JE, Landau B. Intact perception of biological motion in the face of profound spatial deficits: Williams syndrome. Psychol Sci. 2002;13(2):162-7.

Karmiloff-Smith A, Brown JH, Grice S, Paterson S. Dethroning the myth: cognitive dissociations and innate modularity in Williams syndrome. Dev Neuropsychol. 2003;23(12):227-42.

Katayama Y, Tsukiyama T, Tsubokawa T. Thalamic negativity associated with the endogenous late positivity component of cerebral evoked potential (P300): recordings using discriminative aversive conditioning in humans and cats. Brain Res Bull. $1985 ; 14: 223-6$.

Kaufman A, Kaufman N. Kaufman brief intelligence test. 2nd ed. Circle Pines: AGS Publishing; 2004.

Key A, Dove G, Maguire M. Linking brainwaves to the brain: an ERP Primer. Dev Neuropsychol. 2005;27(2):183-215.

Kimchi R. Primacy of wholistic processing and global/local paradigm: a critical review. Psychol Bull. 1992;112:24-38.

Knight RT, Scabini D, Woods DL, Clayworth CC. The effects of lesions of superior temporal gyrus and inferior parietal lobe on temporal and vertex components of the human AEP. Electroencephalogr Clin Neurophysiol. 1988;70:499-508.

Laing E, Butterworth G, Ansari D, Gsödl M, Longhi E, Panagiotaki G, et al. Atypical development of language and social communication in toddlers with Williams syndrome. Dev Sci. 2002;5:233-46.

Lamb M, Robertson L. The processing of hierarchical stimuli: effects of retinal locus locational uncertainty, and stimulus identity. Percept Psychophys. 1988;44:172-81.

Luck S. Multiple mechanisms of visual-spatial attention: recent evidence from human electrophysiology. Behav Brain Res. 1995;71:113-23.

Mangun GR, Hillyard SA, Luck SJ. Electrocortical substrates of visual selective attention. In: Meyer D, Kornblum S, editors. Attention and performance 14: Synergies in experimental psychology, artificial intelligence, and cognitive neuroscience. Cambridge: The MIT Press; 1993. pp. 219-43.

Marr D. Vision: a computational investigation into the human representation and processing of visual information. San Francisco: Freeman; 1982.

Martens M, Wilson S, Reutens D. Williams syndrome: a critical review of the cognitive, behavioral, and neuroanatomical phenotype. J Child Psychol Psychiatry. 2008;49(6):576-608.

Martin M. Local and global processing: the role of sparsity. Mem Cogn. 1979;7:476-84.

Mervis CB, Bertrand J. Developmental relations between cognition and language: evidence from Williams syndrome. In: Adamson LB, Romski MA, editors. Research on communication and language 
disorders: contributions to theories of language development. New York: Brookes; 1997. p. 75-106.

Mervis CB, Klein-Tasman BP. Williams syndrome: cognition, personality, and adaptive behavior. Ment Retard Dev Disabil Res Rev. 2000;6:148-58.

Mervis C, Morris C, Bertrand J, Robinson B. Williams syndrome: findings from an integrated program of research. In: TagerFlusberg H, editor. Neurodevelopmental disorders: contributions to a new framework from the cognitive neurosciences. Cambridge: MIT Press; 1999. pp. 65-110.

Mervis CB, Robinson BF, Bertrand J, Morris CA, Klein-Tasman BP, Armstrong SC. The Williams Syndrome cognitive profile. Brain Cogn. 2000;44:604-28.

Meyer-Lindenberg A, Kohn P, Mervis CB, Kippenhan JS, Olsen RK, Morris CA, et al. Neural basis of genetically determined visuospatial construction deficit in Williams syndrome. Neuron. 2004;43(5):623-31.

Mobbs D, Eckert MA, Menon V, Mills D, Korenberg J, Galaburda $\mathrm{AM}$, et al. Reduced parietal and visual cortical activation during global processing in Williams syndrome. Dev Med Child Neurol. 2007;49(6):433-8.

Nardini M, Atkinson J, Braddick O, Burgess N. Developmental trajectories for spatial frames of reference in Williams syndrome. Dev Sci. 2008;11(4):583-95.

Navon D. Forest before trees: the precedence of global features in visual perception. Cogn Psychol. 1977;9:353-83.

Navon D. Testing a queue hypothesis for the processing of global and local information. J Exp Psychol Gen. 1991;120:173-89.

Navon D, Norman D. Does global precedence really depend on visual angle? J Exp Psychol Hum Percept Perform. 1983;9:955-65.

Neisser U. Cognitive psychology. New York: Appleton-CenturyCrofts; 1967.

Oldfield RC. The assessment and analysis of handedness: the Edinburgh inventory. Neuropsychologia. 1971;9:97-113.

Pani J, Mervis C, Robinson B. Global spatial organization of people with Williams syndrome. Psychol Sci. 1999;10:453-8.

Pellicano E, Gibson L, Maybery M, Durkin K, Badcock DR. Abnormal global processing along the dorsal visual pathway in autism: a possible mechanism for weak visuospatial coherence? Neuropsychologia. 2005;43(7):1044-53.
Pober BR, Dykens EM. Williams syndrome: an overview of medical, cognitive, and behavioral features. Child Adolesc Psychiatr Clin N Am. 1996;5:929-43.

Porter M, Coltheart M. Global and local processing in Williams syndrome, autism, and Down syndrome: perception, attention, and construction. Dev Neuropsychol. 2006;30(3):771-89.

Proverbio AM, Minniti A, Zani A. Electrophysiological evidence of a perceptual precedence of global vs. local visual information. Brain Research:Cognitive Brain Research. 1998;6(4):321-34.

Reiss AL, EckertMA RFE, Karchemskiy A, Kesler S, Chang M, Reynolds MF, et al. An experiment of nature: brain anatomy parallels cognition and behavior in Williams syndrome. $\mathrm{J}$ Neurosci. 2004;24(21):5009-15.

Reiss JE, Hoffman JE, Landau B. Motion processing specialization in Williams syndrome. Vis Res. 2005;45(27):3379-90.

Roalf D, Lowery N, Turetsky BI. Behavioral and physiological findings of gender differences in global-local visual processing. Brain Cogn. 2006;60(1):32-42.

Sarpal D, Buchsbaum BR, Kohn PD, Kippenhan JS, Mervis CB, Morris $\mathrm{CA}$, et al. A genetic model for understanding higher order visual processing: functional interactions of the ventral visual stream in Williams syndrome. Cereb Cortex. 2008;18(10):2402-9.

Spencer K, Dien J, Donchin E. A componential analysis of the ERP elicited by novel events using a dense electrode array. Psychophysiology. 1999;36:409-14.

Stoffer T. The time course of attentional zooming: a comparison of voluntary and involuntary allocation of attention to the levels of compound stimuli. Psychol Res. 1993;56:14-25.

Strømme P, Bjørnstad PG, Ramstad K. Prevalence estimation of Williams syndrome. J Child Neurol. 2002;17(4):269-71.

Tager-Flusberg H, Plesa-Skwerer D, Faja S, Joseph RM. People with Williams syndrome process faces holistically. Cognition. 2003;89 (1):11-24.

Vogel EK, Luck SJ. The visual N1 component as an index of a discrimination process. Psychophysiology. 2000;37:190-203.

Wang P, Doherty S, Rourke S, Bellugi U. Unique profile of visuoperceptual skills in a genetic syndrome. Brain Cogn. 1995;29:54-65.

Ward L. Determinants of attention to local and global features of visual forms. J Exp Psychol Hum Percept Perform. 1982;8:562-81. 